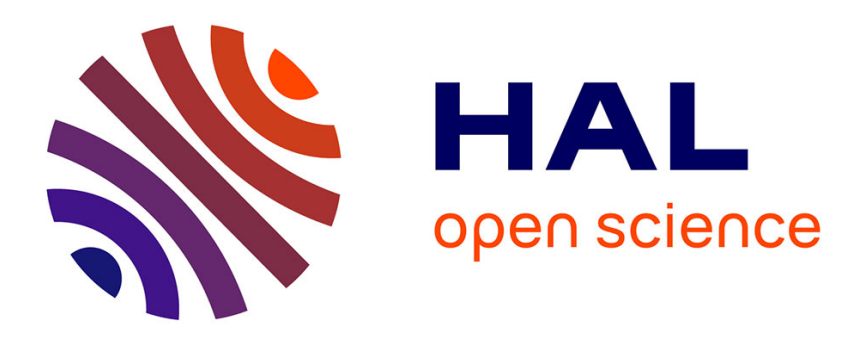

\title{
Une pastorale oubliée: les conseillers agricoles et le progrès
}

Sylvain Brunier, Jacques J. Rémy

\section{To cite this version:}

Sylvain Brunier, Jacques J. Rémy. Une pastorale oubliée : les conseillers agricoles et le progrès. Raison Présente, 2014, 189, pp.21 - 29. 10.3917/rpre.189.0021 . hal-01439267

\section{HAL Id: hal-01439267 https://hal.science/hal-01439267}

Submitted on 19 Jan 2017

HAL is a multi-disciplinary open access archive for the deposit and dissemination of scientific research documents, whether they are published or not. The documents may come from teaching and research institutions in France or abroad, or from public or private research centers.
L'archive ouverte pluridisciplinaire HAL, est destinée au dépôt et à la diffusion de documents scientifiques de niveau recherche, publiés ou non, émanant des établissements d'enseignement et de recherche français ou étrangers, des laboratoires publics ou privés. 


\title{
Une pastorale oubliée : les conseillers agricoles et le progrès
}

\author{
Sylvain Brunier, Jacques Rémy
}

«Après force méditations, Bouvard reconnut qu'il s'était trompé. Son domaine exigeait la grande culture, le système intensif, et il aventura ce qui lui restait de capitaux disponibles ; trente mille francs.

Excité par Pécuchet, il eut le délire de l'engrais. Dans la fosse aux composts furent entassés des branchages, du sang, des boyaux, des plumes, tout ce qu'il pouvait découvrir. Il employa la liqueur belge, le lizier suisse, la lessive, des harengs saurs, du varech, des chiffons, fit venir du guano, tâcha d'en fabriquer, et, poussant jusqu'au bout ses principes, ne tolérait pas qu'on perdît l'urine; il supprima les lieux d'aisances. On apportait dans sa cour des cadavres d'animaux, dont il fumait ses terres. Leurs charognes dépecées parsemaient la campagne. Bouvard souriait au milieu de cette infection. Une pompe installée dans un tombereau crachait du purin sur les récoltes. À ceux qui avaient l'air dégoûté, il disait :

Mais c'est de l'or ! c'est de l'or ! »

Flaubert, Bouvard et Pécuchet

Flaubert semblait douter que « le délire de l'engrais » puisse constituer un véritable progrès ${ }^{1}$. Bouvard s'était fié aux manuels des agronomes pour tirer parti d'un investissement hasardeux, mais n'avait-il pas perdu le sens commun par la même occasion? Pour confronter son expérience avec ses contemporains, la fréquentation des premiers comices agricoles aurait pu s'avérer judicieuse, mais c'est une autre histoire ${ }^{2}$. A la fin du siècle de Flaubert, les services du ministère de l'Agriculture entreprirent de rationaliser l'apprentissage des bonnes pratiques agronomiques en déléguant dans chaque département des ingénieurs dédiés à la vulgarisation du progrès technique ${ }^{3}$. Mais si tous les agriculteurs n'étaient pas, à raison, aussi aventureux que Bouvard et Pécuchet, les résultats demeuraient en-dessous des attentes.

En ce sens, les années qui suivent la Seconde Guerre mondiale marquent une rupture nette avec l'ensemble des expériences précédentes. Des moyens considérables sont débloqués, via les aides du Plan Marshall d'une part, et l'affectation d'une parcelle de l'impôt sur le foncier non bâti aux Chambres d'agriculture d'autre part, permet le développement de leurs services techniques. Surtout, le renouvellement de la vulgarisation agricole passe par l'importation de nouvelles idées : hauts fonctionnaires et responsables syndicaux agricoles découvrent, grâce aux «missions de productivité » vers les États-Unis et l'Europe du Nord-Ouest, les vertus des nouveaux dispositifs d'encadrement combinant, à l'échelle d'une petite région, l'allocation d'aides financières pour inciter les agriculteurs à investir et la mise à disposition permanente d'un conseiller pour contrôler et

1 Pour une réflexion sur les usages de la bibliographie technique employée par Flaubert lors de la rédaction de son ouvrage, voir Gayon Jean, «Agriculture et agronomie dans Bouvard et Pécuchet de Gustave Flaubert », in Littérature, $\mathrm{n}^{\circ} 109,1998$, p. 59-73.

2 Flaubert nous entraîne dans un comice agricole au chapitre 8 de Madame Bovary.

3 Muller Pierre, Le Technocrate et le paysan : essai sur la politique française de modernisation de l'agriculture de 1945 à nos jours, Paris, Éditions ouvrières, 1984. 
encourager ces investissements ${ }^{4}$.

Ce système se généralise au cours des années 1950 et les conseillers agricoles deviennent la cheville ouvrière de la politique de modernisation de l'agriculture française ${ }^{5}$. Souvent fils d'agriculteurs, anciens militants de la Jeunesse agricole catholique pour certains d'entre eux, ils ne souhaitaient pas rester de simples techniciens chargés de faire appliquer sur le terrain les dispositions décidés d'en haut pour accroître la productivité de «la ferme France ». Ils étaient profondément convaincus de l'intérêt de leur travail pour assurer la promotion des exploitations familiales, c'est-à-dire faire entrer le monde agricole de plain pied dans la modernité. Avoir foi dans l'avenir signifiait pour eux qu'il était possible de faire advenir un monde meilleur, où la modernité conjuguerait une plus grande productivité avec l'épanouissement de la personne et l'accession aux standards de vie urbains (la parité réclamée par les jeunes agriculteurs).

Rétrospectivement, leur foi dans le progrès pourrait apparaître quelque peu naïve. La critique du productivisme et la remise en question du récit dominant sur les Trente glorieuses ont discrédité l'idéal modernisateur, entendu comme un élan unanime vers le progrès, pour mettre en lumière les conflits et les renoncements que ce discours politique avait pu recouvrir ${ }^{6}$. Dans les conversations courantes, la déploration de la destruction des savoirs et savoir-faire vernaculaires a remplacé la fascination pour le perfectionnement des procédés techniques. La ligne générale d'Eisenstein et son ode au progrès appartiennent à un passé révolu, les Profils paysans de Depardon empreints de nostalgie sont plus raccords avec notre époque ${ }^{7}$. Alors que les Jeunes agriculteurs des années 1950 étaient loués pour avoir rompu avec la « routine» dans laquelle étaient enfermés leurs parents, on leur reproche désormais d'avoir sacrifié l'authenticité de leur mode de vie pour satisfaire leurs aspirations matérielles immédiates.

Le progrès ne fait plus recette mais les lectures téléologiques demeurent. Remettre les conseillers agricoles au cœur de l'histoire de la modernisation des campagnes après-guerre, c'est faire un pas de côté par rapport au mythe des agriculteurs qui auraient, seuls, "pris leur destin en main » pour «monter dans le train du progrès ». Ils étaient bien au cœur d'un projet politique, dans lequel leurs organisations professionnelles ont joué un rôle décisif. Mais c'est aussi faire un pas de côté par rapport à un travail de démystification critique qui traiterait l'amour du progrès comme un vernis illusoire apposé pour faire oublier la violence des transformations objectives des structures économiques. Les conseillers agricoles partageaient la même croyance que les responsables syndicaux agricoles : le progrès technique devait permettre à l'agriculteur et à sa famille de prendre leur place au sein de la société, eux qui avaient vécu l'urbanisation et l'industrialisation croissantes comme un lent déclassement, eux qui avaient la hantise de la dépopulation du monde rural, eux qui n'acceptaient pas, ou plus, de rester à l'écart du mouvement général de la société.

\section{La tâche}

On ne comprend pas grand chose à la séquence qui suit la Libération dans les campagnes si on refuse de prendre au sérieux l'émotion qui a guidé l'action des premiers conseillers agricoles : leur amour du progrès ne doit pas s'entendre comme une forme de dévotion à la Technique toutepuissante mais plutôt comme une aspiration à une reconnaissance des qualités morales des familles

4 Les missions de productivité concernaient l'ensemble des secteurs de l'économie. Voir Barjot Dominique, Catching up with America : Productivity missions and the diffusion of American Economic and Technological Influence after the Second World War, Paris, PUPS, 2002.

5 Brunier Sylvain, Conseillers et conseillères agricoles en France. L'amour du Progrès aux temps de la "révolution silencieuse" (1945-1983), thèse d'histoire, Université de Grenoble, 2012.

6 Bessis Céline, Topçu Sezin et Bonneuil Christophe (dir.), Une autre histoire des Trente Glorieuses : modernisation, contestations et pollutions dans la France d'après-guerre, Paris, La Découverte, 2013.

7 Bessière Céline, Bruneau Ivan, «La Vie moderne : la beauté de la mort paysanne », in Revue de Synthèse, Tome 136, 6e série, $\mathrm{n}^{\circ} 3,2011$, p. 448-454. 
agricoles et de leur contribution à l'effort national de reconstruction. Cette soif de reconnaissance est au cœur du travail de mobilisation réalisé par la Jeunesse agricole catholique : en organisant des manifestations festives, en publiant des bulletins d'information, en structurant des activités culturelles ou sportives, il s'agit de montrer en actes que les agriculteurs sont partie prenante des débats politique et des évolutions sociétales.

Les militants de la JAC investissent progressivement l'ensemble des organisations professionnelles agricoles, d'abord le syndicalisme jeune (le CNJA) dans les années 1950, puis le syndicalisme aîné (la FNSEA) à partir des années 1960. Ils sont également à l'origine de l'essor du mouvement des Centre d'études techniques agricoles (CETA), petits groupes d'agriculteurs qui entreprennent de mettre en commun leurs réflexions en s'appuyant sur les conseils d'un technicien. Dans les Chambres d'agriculture, cette nouvelle génération de responsables syndicaux remplace peu à peu les anciens notables ${ }^{8}$. Évinçant les services du ministère de l'Agriculture des opérations de vulgarisation sur le terrain, ils obtiennent de constituer de véritables services techniques au sein des Chambres, et multiplient les embauches de conseillers qui partagent leur conception du progrès.

Ce travail de mobilisation oblige l'ensemble des acteurs de la politique agricole à redéfinir leur manière d'envisager le développement agricole. Par exemple, les instituteurs qui réalisaient les cours post-scolaires agricoles reprennent la formule des petits groupes autonomes et lancent les Centres d'initiative pour valoriser l'agriculture et le milieu rural (CIVAM). Ces structures laïques s'implantent essentiellement dans les territoires où l'Action catholique est moins présente. Elles participent du bouillonnement politique propre au monde rural après-guerre, l'ensemble des initiatives convergeant autour des idées de valorisation, de promotion ou d'émancipation. L'emploi du mot «développement », qui s'impose dans les textes officiels à partir de 1966, doit souligner ainsi la rupture avec l'ancienne manière de concevoir la vulgarisation du progrès technique et la reconnaissance de l'implication directe des agriculteurs et de leurs familles dans le processus de modernisation.

Le travail des conseillers agricoles demeure ambivalent puisqu'ils sont chargés à la fois de faire «descendre » un certain nombre de prescriptions techniques ou financières - ils sont les instruments d'une politique publique qui associe l'État et la profession - mais ils ont aussi vocation à faire « remonter » les doléances et les souhaits des agriculteurs engagés dans la modernisation ils sont leurs interlocuteurs directs, ceux en qui ils placent toute ou partie de leur confiance lorsqu'il s'agit de s'assurer de la pertinence d'un investissement ou de structurer une initiative collective. L'identité professionnelle du conseiller est le produit de cet équilibre instable. S'il rompt avec l'une ou l'autre de ses fonctions, il sera accusé d'avoir mené un double jeu, d'être un agent manipulateur au service d'une politique sans véritable considération pour son interlocuteur s'il se fait trop directif, d'être un potentat local ayant tiré parti de son autorité morale sur les agriculteurs pour usurper une légitimité politique s'il devient trop indépendant.

La croyance dans le progrès est le ciment idéologique qui permet au conseiller de tenir sa position. C'est parce qu'il partage au moins une partie des aspirations des agriculteurs avec lesquels il travaille qu'il est en mesure de se faire écouter. Recrutés notamment en fonction de leurs origines familiales, leurs employeurs considérant qu'il leur sera plus facile d'exercer ce métier s'ils ont une connaissance pratique du «milieu », les jeunes conseillers suscitent pourtant une grande méfiance lors de leurs premières visites. C'est qu'aussi familiers soient-ils, ou veulent-ils être, des problèmes des agriculteurs, ils sont perçus d'abord comme les représentants d'une autorité extérieur, fusse-telle celle du syndicat ou de la Chambre. Dans un monde où les relations interpersonnelles demeurent la première ressource cognitive, le métier de conseiller reste à inventer. Ne serait-ce que pour être autorisés à franchir le pas de la porte dans certaines fermes, et a fortiori pour que leur parole devienne sinon crédible, du moins audible, ils doivent faire la preuve qu'ils sont dignes de

\footnotetext{
8 Atrux-Tallau Mélanie, Histoire sociale d'un corps intermédiaire : l'Assemblée permanente des chambres
} d'agriculture (1924-1974), thèse d'histoire, Université Lyon 2, 2010. 
confiance 9 .

Les deux vertus cardinales du conseiller sont le dévouement et le sens de la ruse, au sens de la mètis des Grecs, c'est-à-dire une forme d'intelligence qu'on pourrait rapprocher de l'art de naviguer entre les récifs ${ }^{10}$. Par dévouement, il faut entendre sa capacité à dépasser son rôle de technicien ou d'animateur en montrant à quel point il est investi dans son métier. En sillonnant les routes enneigées, en organisant les réunions d'information le dimanche après-midi ou en visitant les exploitations à la nuit tombée pour établir un plan d'investissement, il s'agit non seulement de s'adapter au rythme de travail des agriculteurs en profitant des temps morts pour capter leur attention, mais aussi de démontrer qu'on croit dans son travail au point de ne pas compter ses heures, de sacrifier son confort et une partie de sa vie de famille. Les responsables des organisations professionnelles agricoles appréciaient d'ailleurs que le conseiller, et davantage encore, la conseillère, reste célibataire afin de se consacrer entièrement à son travail.

Mais le conseiller ne doit pas seulement être un prosélyte zélé des dernières innovations en matière de fertilisation ou de machinisme. Il acquiert le respect des agriculteurs en montrant qu'il est capable de contourner les règles administratives et les tentations commerciales. Les conseillers mettent un point d'honneur à se distinguer des fonctionnaires du ministère de l'Agriculture, critiqués pour la pesanteur de leurs procédures, et des représentants de commerce, dont ils dénoncent la propension à vouloir les soudoyer pour qu'ils fassent la promotion de tel ou tel de leur produit. Le titre de gloire d'un conseiller c'est par exemple d'avoir obtenu d'un commandant d'une base de l'armée de l'air que ses hélicoptères mettent leurs manœuvres à profit pour héliporter le matériel nécessaire aux alpagistes de son secteur. Si la légitimité première du conseiller est bien son expertise technique, ce n'est qu'en montrant qu'il est capable de louvoyer entre les contraintes pour sauvegarder les intérêts de ses interlocuteurs, au mépris des risques et des normes, qu'il peut véritablement exercer son métier.

Tel que le conçoivent les conseillers, le progrès renvoie à la promesse d'un avenir meilleur dans lequel le travail et l'investissement des agriculteurs engagés dans la modernisation seront reconnus à leur juste valeur. Ces derniers pourront enfin avoir voix au chapitre dans les grands choix politiques et sociaux, sans être pour autant obligés de renoncer à leur spécificité culturelle et morale. Nombre de ces conseillers sont d'autant mieux placés pour faire cette promesse, pourtant difficile à tenir, qu'ils se sont eux-mêmes engagés dans ce métier avec l'espoir qu'ils pourraient échapper au mode de vie de leurs parents sans jamais rompre complètement avec leurs attaches. S'ils ont tenu jusqu'à la fin de leur carrière, malgré les crises redondantes de la politique de développement agricole à partir des années 1970, ils ne sont pas parvenus à transmettre leur enthousiasme et leur croyance dans le progrès.

\section{Exit le fantôme}

Après les années 1980, cette façon d'exercer le métier de conseiller agricole a fait place à d'autres conceptions, à d'autres pratiques, alors même que le nombre d'intervenants dans le secteur para-agricole n'a jamais été aussi élevé ${ }^{11}$. Les nouveaux techniciens se distinguent de leurs prédécesseurs précisément en ce qu'ils ne font plus directement référence à une notion générale de progrès. Désormais, l'évaluation des performances prime sur l'impulsion du mouvement, suivant en cela le mouvement général de la société. Le projet modernisateur ébauché dans les années 1950 n'a pas connu de véritable renouvellement et les générations qui l'ont porté initialement ont peu à peu

9 Rémy, Jacques, «Entre familles, marchés et politiques publiques : le conseiller agricole », in Revue de l'INRA, ${ }^{\circ}$ 127, été 2006, p. 38-40.

10 Détienne Marcel et Vernant Jean-Pierre, Les ruses de l'intelligence : la mètis des Grecs, Paris, Flammarion, 1974.

11 Compagnone Claude, Auricoste Caroline et Lémery Bruno (dir.), Conseil et développement en agriculture : quelles nouvelles pratiques?, Dijon/Versailles, Éducagri/ Quae, 2009. 
abandonné leurs responsabilités. Dans ce contexte, les conseillers ne sont pas parvenus à transmettre les principes de leur éthique professionnelle, et de là, à continuer de faire vivre leur idéal de progrès.

Dans les récits que les anciens conseillers font de leur carrière, des anecdotes illustrent le décalage entre le futur retraité et le nouvel entrant, ici l'organisation d'une importante réunion de responsables agricoles se terminant en fiasco du fait de l'imprévoyance du remplaçant, là le renoncement à tenir une réunion dans un village de montagne du fait des mauvaises conditions météorologiques, et partout le sentiment que la qualité des relations humaines avec les agriculteurs est négligée. Au-delà de ce que la sévérité de ces jugements doit à la cessation d'activité, difficile à accepter pour celles et ceux qui se considéraient comme des activistes du développement agricole, les regrets sont fondés sur le constat de l'écart irréductible entre ce qu'était leur état d'esprit, construit autour de la notion centrale de service des agriculteurs, et ce qu'ils perçoivent des nouveaux embauchés, qui ont une formation scolaire supérieure à la leur mais n'entendent pas s'investir dans leur travail de la même manière, préférant instaurer un rapport plus sécurisant de salarié à employeur.

Tenus de rester en retrait par rapport à leurs employeurs tout au long de leur carrière et privés des successeurs qui auraient pu relayer leur mémoire, les conseillers d'alors se trouvent cantonnés à une place marginale dans les récits de la modernisation agricole d'après-guerre. Amputée de la sorte, cette histoire ne permet pas de ressaisir les ressorts de la mobilisation des agriculteurs. Le progrès est réduit à une accumulation d'innovations techniques suivant une logique fordiste, et la modernisation à une entreprise d'adaptation des structures de production agricoles aux exigences de l'économie de marché. Effacer le rôle complexe que les conseillers ont joué dans cette histoire, c'est méconnaître la puissance des aspirations morales du monde agricole qui soustendaient leur élan vers le progrès ${ }^{12}$. La relation que le conseiller nouait avec «ses » agriculteurs dépassait de loin les seules questions de productivité et de gestion, elle pouvait servir de point d'appui pour les plus dominés, et notamment les femmes, pour affirmer leur existence et tenter de prendre leur place, au sein de la famille, de l'exploitation, de la vie politique locale.

Pour autant, dès les années 1970, la critique du productivisme avait bien souligné l'impasse dans laquelle les conseillers avaient conduit les agriculteurs les plus fragiles. Et plus encore, lorsque certains bons élèves de la politique de développement se trouvèrent à leur tour en difficulté, c'est toute la crédibilité de l'édifice qui s'effondra. Le progrès pouvait désormais apparaitre comme une espérance illusoire qui avait nourri les pires excès. Il ne s'agit pas ici de minimiser l'ampleur des destructions environnementales et sociales qui sont les conséquences directes de l'intensification incessante de la production. Mais en réduisant l'ensemble du processus de modernisation à une course dévastatrice, les critiques de la modernisation ont paradoxalement manqué leur cible. En refusant de prendre en compte la dimension morale de l'élan modernisateur, l'aspiration des agriculteurs à plus grande reconnaissance de la part du corps social, pour ne retenir que les effets les plus destructeurs de leurs initiatives, la critique du progrès s'est superposée à la critique de l'inefficacité de la politique de développement, portée par certaines organisations agricoles (coopératives et crédit au premier chef) et administrations, visant le renforcement des performances techniques et économiques des exploitations en-dehors de toute considération pour les idées de promotion ou d'émancipation. Cette double critique a cisaillé la notion même de progrès, rendant illisible le rapport que les conseillers agricoles entretenaient avec leur travail.

La notion de progrès n'est plus guère mobilisée aujourd'hui dans la politique agricole. Les travaux de déconstruction ont pris le dessus sur les discours enchantés. Mais cela n'a pas forcément eu l'effet escompté. Les logiques de sélection économique des exploitations, de spécialisation et d'intensification des modes de production, se sont poursuivies jusqu'aujourd'hui, bien que privées du

12 Didier Fassin et Jean-Sébastien Eideliman (dir.), Economies morales contemporaines, Paris, La Découverte, 2012. 
référentiel idéologique qui les justifiait dans les décennies d'après-guerre. Les institutions créées pour soutenir la politique de développement ont continué de fonctionner, mais le mouvement social des agriculteurs sur lequel elles reposaient s'est largement étiolé. On ne peut s'empêcher de penser que les opposants à l'industrialisation de l'agriculture, qui n'a jamais été aussi avancée bien que des niches spécifiques aient été créées autour des produits de terroir, auraient tout intérêt à se réapproprier la notion de progrès et le potentiel de mobilisation qu'elle enferme. En ce sens, l'histoire des conseillers agricoles nous rappelle combien il importe de disposer d'un corps de spécialistes convaincus de l'intérêt de leur mission lorsqu'il s'agit d'opérer un tournant politique, économique et social majeur, hier l'accroissement spectaculaire de la productivité, aujourd'hui peutêtre, l'environnementalisation et l'agro-écologisation de la politique agricole. 